GROWTH KINETICS OF GRAIN-BOUNDARY INDUCED MELTING: A MOLECULAR-DYNAMICS STUDY*



CONF-8811147--2

S. R. PHILLPOT, J. F. LUTSKO, D. WOLF, and S. YIP*: Materials Science Division

DE89 009855

Argonne National Laboratory Argonne IL 60439.

**Permanent address: Department of Nuclear Engineering, Massachusetts Institute of Technology, Cambridge MA 02139.

\title{
DECEMBER 1988
}

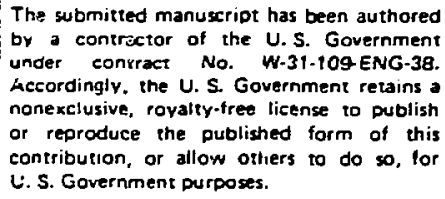

\section{DISCLAIMER}

This report was prepared as an account of work sponsored by an agency of the United States Government. Neither the United States Government nor any agency thereof, nor any of their employees, makes any warranty, express or implied, or assumes any legal liability or responsibility for the accuracy, completeness, or usefulness of any information, apparatus, product, or process disclosed, or represents that its use would not infringe privately owned rights. Reference herein to any specific commercial product, process, or service by trade name, trademark. manufacturer, or otherwise daes not nesessarily constitute or imply its endorsement, recommendation, or favoring by th: United States Government or any agency thereof. : ihe views and opinions of authors expressed herein do not necessarily state or reflect those of the United States Government or any agency thereof.

*Work supported by the U.S. Department of Energy, BES Materials Sciences, under Contract W-31-109-Eng-38.

Submitted to MRS Symposium T on "Atomic Scale Calculations in Materials Science", Boston, MA, Nov. 28 - Dec. 3, 1988.

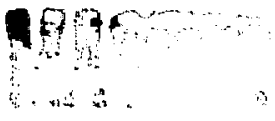


S. R. PHILLPOT, J. F. LUTSKO, D. WOLF and S. YIP*

Materials Science Division, Argonne National Laboratory, Argonne IL 60439.

\section{ABSTRACT}

The growth kinetics of melting nucleated at a high-angle twist boundary in silicon are investigated using molecular dynamics. Melting is found to be a two-stage process. In the first stage order is lost within a single plane at the interface and the density of the solid increases to that of the liquid. In the second stage the atomic coordination changes and an isotropic liquid is formed.

\section{INTRODUCTION}

Melting is generally nucleated at a lattice defect, such as a grain boundary $(\mathrm{GB})$, free surface or dislocation $[1,2,3]$, and in most materials superheating is only possible under rather unusual conditions [3]. Recent molecular-dynamics studies in both silicon [4] and metals [5] are consistent with these experimental observations in that they have shown that in the presence of a localized, extended lattice defect superheating is not possible and that melting is nucleated at the lattice defect at the thermodynamic melting temperature.

Here, we focus on examining details of the melting mechanism, after nucleation of the liquid at a high-angle twist grain boundary in silicon [4]. The Stillinger-Weber potential [6] used in this study has proved successful in recent studies of the bulk crystal $[6,7]$, the liquid [7], the crystal-1iquid interface $[8,9]$ and grain boundaries [10].

\section{GRAIN-BOUNDARY GEOMETRY AND CHARACTERIZATION OF DISORDERING AND MELTING}

The GB choser! for our investigation is the so-called $\Sigma 11$ (110) highangle twist boundary. This boundary on the (110) plane is obtained by rotation about [110], by an angle of $\theta=50.48^{\circ}$, of two perfect semicrystals with (110) faces. The area of the rectangular unit cell of this coherent interface is $\Sigma=11$ times greater than that of the corresponding primitive planar unit cell $(\Sigma=1)$ on the (110) plane; each lattice plane contains 22 atoms. Our computational cell is chosen to contain 32 (110) planes, for a total of 704 atoms. Two-dimensionally periodic border conditions, described in detail elsewhere [11], are applied parallel to the boundary $(x-y)$ plane. The lattice parameter in the $x-y$ plane is fixed to trat of the ideal crystal at the temperature simulated. In the $z$-direction ( 2 |l [110]) the simulation cell is embedded between two semi-infinite perfectcrystal blocks which, during the simulation, are allowed to move as rigid units thus allowing both GB sliding and volume expansion at the interface [11]. The zero-temperature structure and energy of this boundary were investigated in Ref. 10. 
To monitor the inhomogeneity of the bicrystal in the z-direction, the system is subdivided into 32 slices; initially every slice thus contains one (110) plane. In every slice the instantaneous values of the potential energy, temperature and mean-squared displacement (msd) are monitored. The total number of defected atoms, $\mathbb{N}_{\text {def, }}$ in the system is also calculated. An atom is considered defected if its number of nearest neighbors is different from that in an ideal crystal. As a merely semi-quantitative measure we define the nearest-neighbor shell to end half way between the ideal-crystal first- and second-nearest-neighbor distances. Thus, any disordering or melting of the system, and subsequent coordination change, will be apparent as an increase in $\mathrm{N}_{\text {def }}$.

To investigate the breakdown of the crystalline order upon melting we also monitor the square of the magnitude of the static structure factor, $S(k)$ (which for brevity we simply denote $S^{2}(k)$ ). For the overall $S^{2}(k)$ all atoms in the simulation cell are included, whereas for a planar structure factor, $S_{p}^{2}(k)$, only atoms in a given lattice plane are considered. For an ideal crystal at zero temperature, $S_{p}^{2}(\underline{k})$ equals unity for any wave vector, $k$, which is a reciprocal lattice vector in the (110) plane. By contrast, in the liquid state (without long-range order in (110) plane), $\mathrm{S}_{\mathrm{p}}^{2}(\mathrm{k})$ fluctuates near zero. As the two halves of the bicrystal are rotated with respect to each other about the GB-plane normal, two different wave vectors, $\underline{k}_{1}$ and $\underline{k}_{2}$ are required, each corresponding to a principal direction in one half of the bicrystal.

\section{GROWTH KINETICS OF GRAIN-BOUNDARY INDUCED MELTING}

Here we consider the results of bicrystal simulations performed at four temperatures: $1800 \mathrm{~K}, 1900 \mathrm{~K}, 2000 \mathrm{~K}$ arid $2100 \mathrm{~K}$, all of which are above the thermodynamic melting temperature, $1691 \pm 20 \mathrm{~K}[7]$, but below the temperature to which a perfect crystal can be superheated without nucleating the liquid $[4,7]$. At the beginning of each simulation run particles are given random velocities corresponding to a temperature of $1200 \mathrm{~K}$. To reach the desired simulation temperature the system is then heated by $100 \mathrm{~K}$ every 200 time steps. As we are primarily interesied in phase transitions which involve a latent heat, a thermostat is applied by rescaling the particle velocities. In all the simulations the time step is $1.15 \cdot 10^{-15}$ seconds. Because we find qualitatively the same behavior at all these temperatures, in Figs. 1-4 only results obtained at $\mathrm{T}=1900 \mathrm{~K}$ are summarized.

Figure 1 shows the instantaneous planar structure factors after 8600 and 17600 time steps for the $1900 \mathrm{~K}$ simulation. They illustrate the spreading of a disordered region, initiated at the GB, into the two semicrystals. Figure 2 shows that the width of this disordered region, defined as the number of planes in which both structure factors have a value of less than 0.5, increases approximately linearly in time, over the entire sim.llation interval. From the rate of increase in this width a velocity of disordering for each of the two disordered-crystalline interfaces of $v_{W}(1900 \mathrm{~K})=39 \pm 5 \mathrm{~m} / \mathrm{s}$ is found.

Knowing the behavior of $S_{p}^{2}(k)$ is not sufficient to tell us whether the disordered region is liquid, disordered but crystalline, or amorphous. One can look to the atomic mobility, as measured by the msd, to aid in making this distinction. We find [4] that the msd of two slices taken from the center of the disordered region attains a linear behavior that is characteristic of diffusive motion. Based on these data, we determine the 


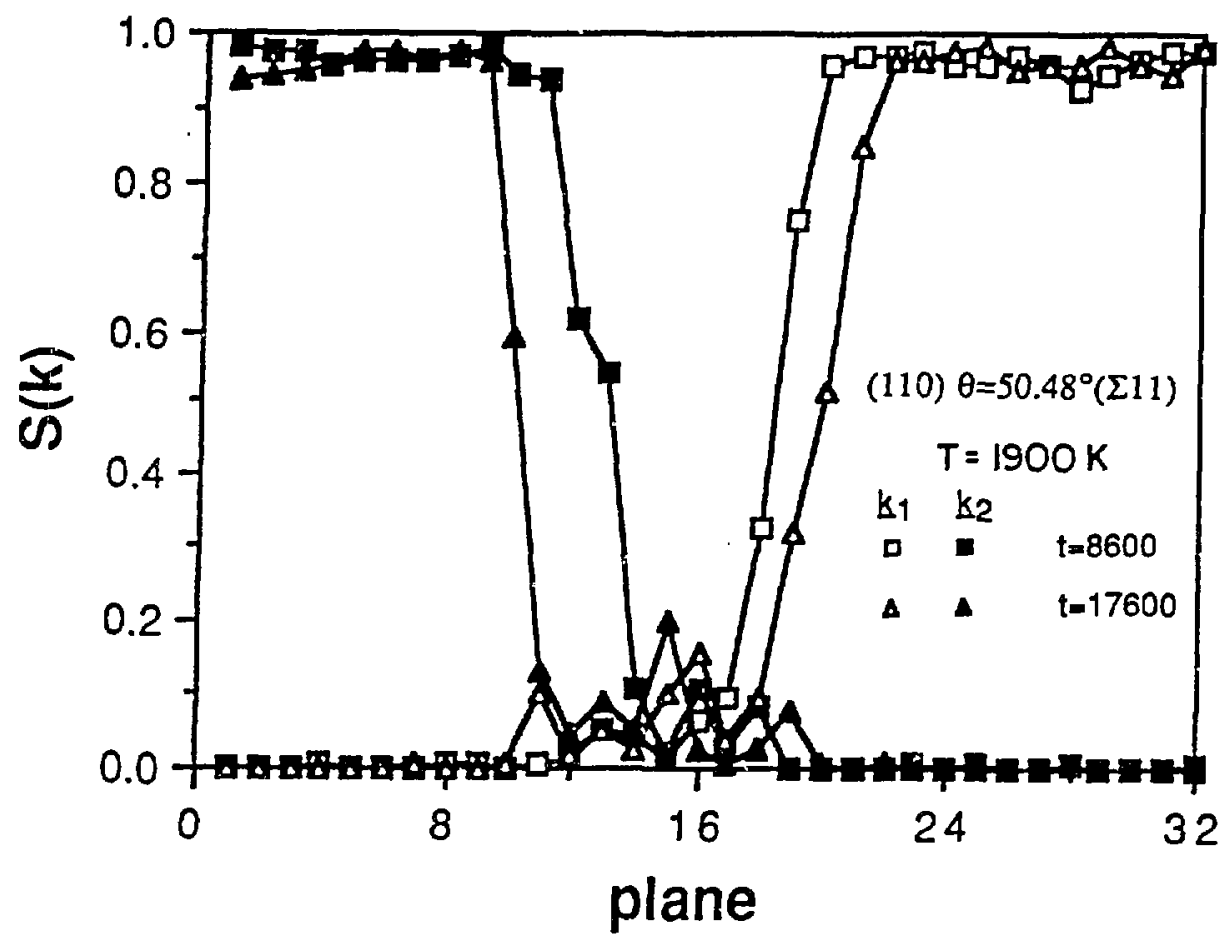

Fig. 1. Instantaneous values of $s_{p}^{2}\left(k_{1}\right)$ and $s_{p}^{2}\left(k_{2}\right)$ for the 32 slices parallel to the (110) $\theta=50.48^{\circ}$ ( $(11) \mathrm{GB}$ after 8600 (squares) and 17600 time steps (triangles) at $1900 \mathrm{~K}$.

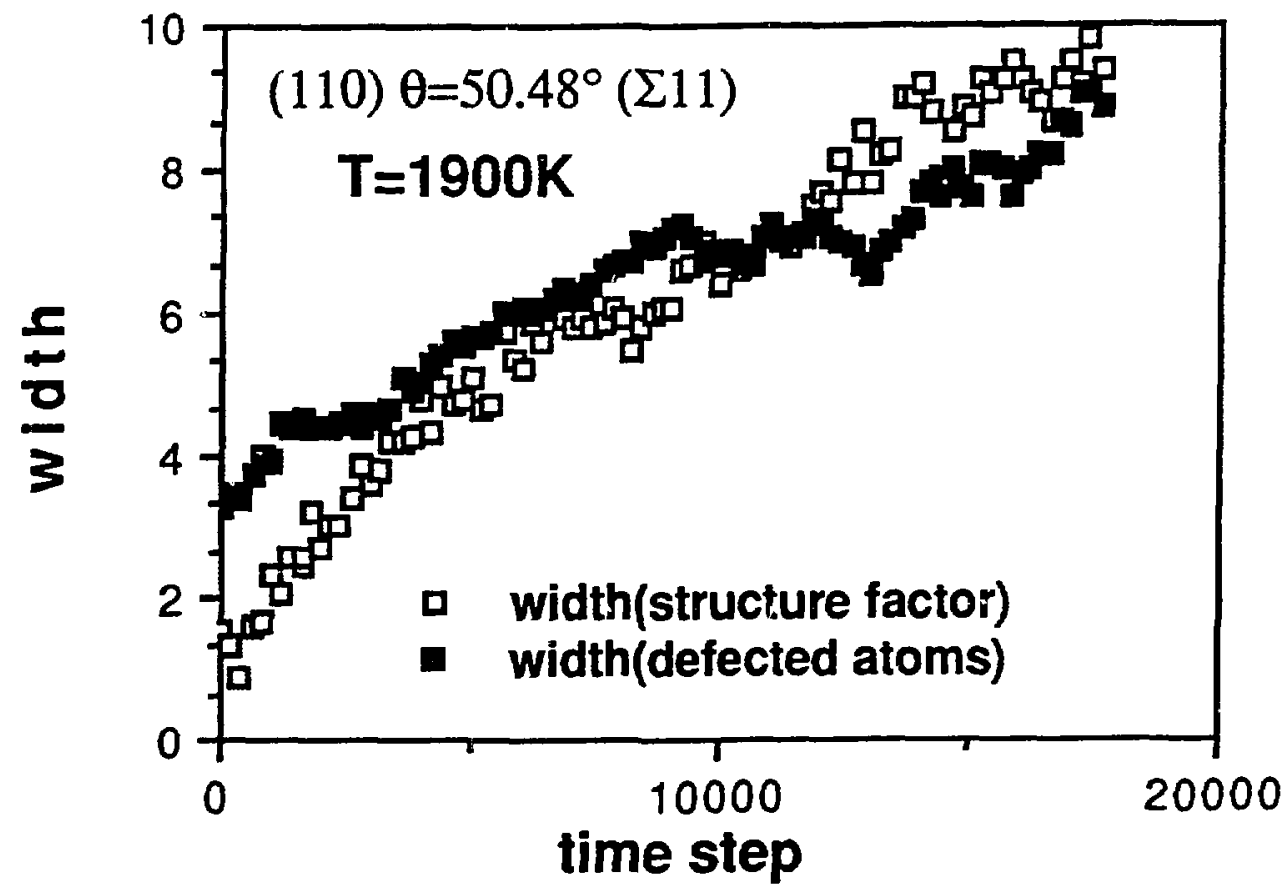

Fig. 2. Instantaneous width, in lattice planes, of the disordered region as a function of time at $1900 \mathrm{~K}$ as calculated from the structure factor (open squares) and from the number of defected atoms (solid squares). 
diffusion constant to be $\approx 0.7 \cdot 10^{-8} \mathrm{~m}^{2} / \mathrm{s}$. This is about the same as the value found for the liquid at this temperature [7]. Thus the disordered region is indeed 1 iquid.

The number of defected atoms, $\mathrm{N}_{\mathrm{def}}$, provides another way of characterizing the disordering of the system, from which we can also obtain a measure of the width of the liquid region. As shown in Eig. 2, this quantity also increases approximately linearly with time. From these data we extract a propagation velocity of $v_{\text {def }}(1900 \mathrm{~K})=28 \pm 5 \mathrm{~m} / \mathrm{s}$.

Table I compares $v_{W}$ and $v_{\text {def }}$ at the four highest temperatures. Significantly, as discussed in detail elsewhere [4], $v_{w}$ and $v_{\text {def }}$ extrapolate to zero velocity at temperatures that are statistically identical both to

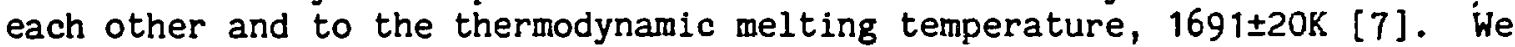
conclude, therefore, that the propagation of the solid-liquid interface is due to ordinary thermodynamic melting of the bicrystal.

It is clear from Table I that $v_{w}$ is significantly greater than $v_{\text {def }}$ at the two higher temperatures. This difference suggests that one may obtain some insight about the mechanism of melting from considering the simulation data in more detail. Three pertinent observations can be summarized as follows:

Table I

Propagation velocity in $\mathrm{m} / \mathrm{s}$ of the two solid-liquid interfaces for melting nucleated at a GB, as determined from an analysis of the GB width $\left(v_{W}\right)$, from the change in density of the system $\left(v_{h}\right)$, and from the number of defected atoms $\left(v_{\text {def }}\right)$.

$1800 \mathrm{~K} \quad 1900 \mathrm{~K} 2000 \mathrm{~K} 2100 \mathrm{~K}$

\begin{tabular}{llllc}
\hline$v_{w}$ & $23 \pm 4$ & $39 \pm 5$ & $83 \pm 10$ & $131 \pm 7$ \\
$v_{h}$ & $24 \pm 6$ & $36 \pm 11$ & $84 \pm 11$ & $127 \pm 15$ \\
$v_{\text {def }}$ & $18 \pm 9$ & $28 \pm 5$ & $52 \pm 7$ & $76 \pm 9$ \\
\hline
\end{tabular}

(i) Figure 3 shows the time dependence of the in-plane and out-ofplane msds for a single slice in the center of the bicrystal. It can be seen that the in-plane mobility increases first, and it is only after about 5000 time steps that atoms begin to move significantly out of the plane. Beyond $\approx 10000$ time steps the slopes of the two curves are similar, an indication that the liquid is becoming fully isotropic.

(ii) Because silicon contracts on melting, the length of the computational cell decreases with time. This decrease is approximately linear in time, as is shown in Fig. 4 for the $1900 \mathrm{~K}$ simulation. Making the reasonahle assumption that the entire density change produced on melting an ideal crystal under three-dimensionally periodic border conditions at zero pressure is reflected in a corresponding density change upon melting of the bicrystal, and using the known densities of the crystal and liquid [7], a further interface velocity, $v_{h}$, can be calculated. We find $v_{h}(1900 \mathrm{~K})=36 \pm 11$ 
$\mathrm{m} / \mathrm{s}$. We note in Table I that, within the error bars, $v_{h}$ is equal to $v_{w}$ and significantly larger than $v_{\text {def }}$ at the two higher temperatures.

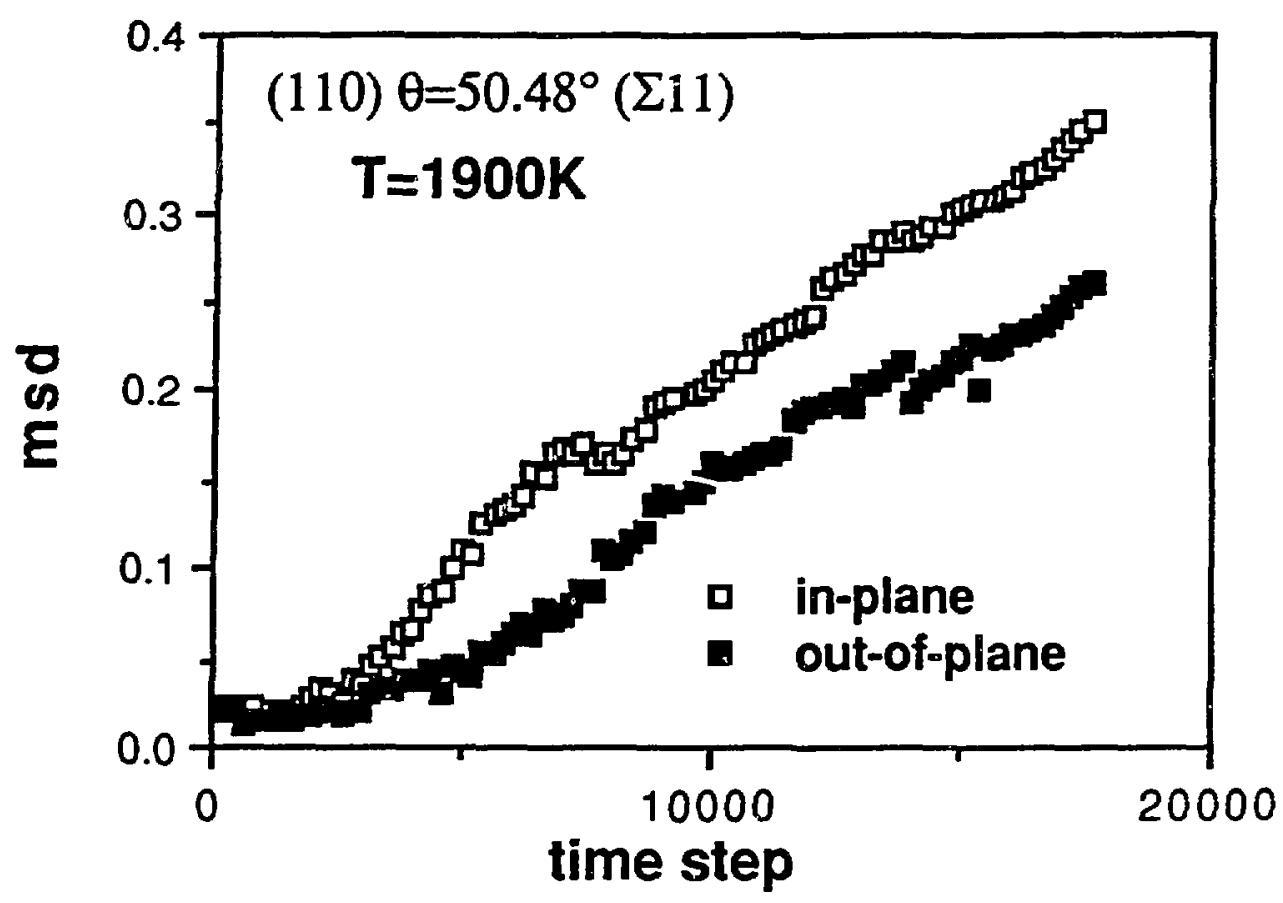

Fig. 3. Mean-squared displacement, in lattice parameters squared, parallel to (open squares) and perpendicular to the GB plane (solid squares) for a slice at the center of the liquid region as a function of time at $1900 \mathrm{~h}$.

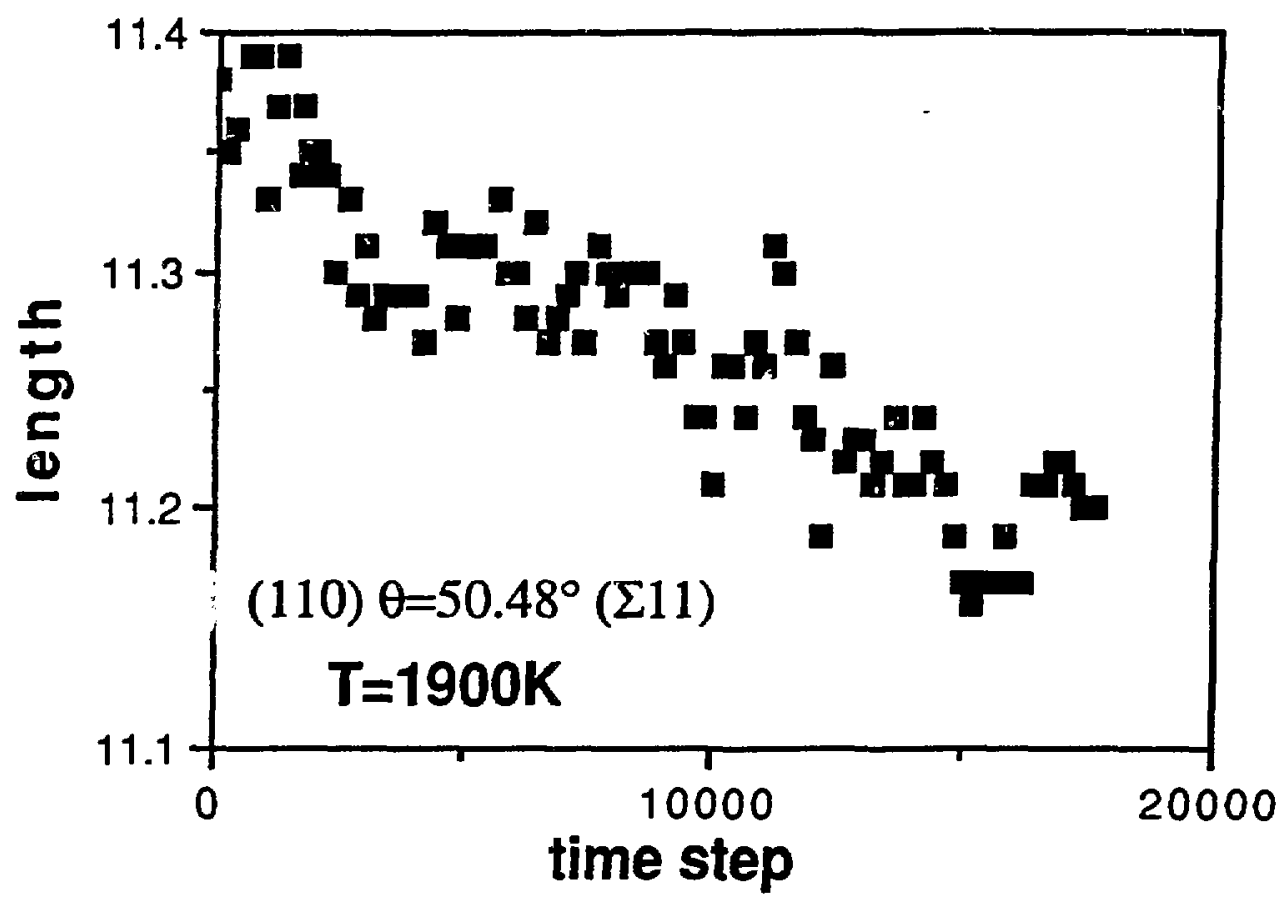

Fig. 4. Length of the bicrystal computational cell in the z-direction (parallel to the GB-plane normal), as a function of time at $1900 \mathrm{~K}$. The length of the cell at $T=O K$ is $11.36 a_{0} \cdot\left(a_{0}\right.$ is the $T=0$ lattice parameter.) 
(iii) The inequality between $v_{y}$ and $v_{\text {def }}$ seems to arise from the different degrees of disorder probed by the planar structure factor and the number of defected atoms. Because the planar structure factor is sensitive to even small deviations from ideal-crystal order, $v_{w}$ may be expected to be larger than $v_{\text {def }}$ which reflects the greater degree of disorder associated with a change of coordination.

\section{DISCUSSION}

Based on the above observations, we envisage the melting of a plane of atoms at the interface as taking place by a two-stage process. In the first stage atoms move only in the plane, as evidenced by the increase in the in-plane msd. This in-plane motion is also reflected in a strong decrease in the planar structure factor. However, although the density of the system increases to that of the liquid, this in-plane atomic motion is apparently insufficient to change the cordination. In the second stage there is also significant out-of-plane mobility and the atomic coordination changes to produce a fully isotropic liquid state.

\section{ACKNOWLEDGMENTS}

We are indebted to Prof. H. Gleiter for several helpful suggestions and discussions. This work was supported by U.S. Department of Energy, BES-Materials Science, under Contract W-31-109-Eng-38. The authors wish to acknowledge a grant of computer time at the Energy Research CRAY XMP at the Magnetic Fusion Computational Center at Livermore.

\section{REFERENCES}

1. R. L. Cormia, J. D. Mackenzie, and D. Turnbull, J. Appl. Phys. 34 , 2239 (1963).

2. R. W. Cahn, Nature 323, 668 (1986).

3. J. Daeges, H. Gleiter, and J. H. Perepezko, Phys. Lett. A119, 79 $(1986)$.

4. S. R. Phillpot, J. F. Lutsko, D. Wolf and S. Yip, submitted to Phys. Rev. B.

5. J. F. Lutsko, D. Wolf, S. R. Phillpot and S. Yip, submitted to Phys. Rev. B.

6. F. H. Stillinger and T. A. Weber, Phys. Rev. B31, 5262 (1985).

7. J. Q. Broughton and X. P. Li, Phys. Rev. B $\underline{3} 5, \overline{9120}$ ( 1987 ).

8. U. Landman, W. D. Leudtke, R. Barnett, C. L. Cleveland, M. W. Ribarsky, E. Arnold, S. Ramesh, H. Baumgart, A. Martinez and B. Kahn, Phys. Rev. Lett. 56, 155 (1986).

9. U. Landman, W. D. Luedtke, M. W. Ribarsky, R. N. Barnett, and C. L. Cleveland, Phys. Rev. B37, 4637 (1988); W. D. Luedtke, U. Landman, M. W. Ribarsky, R. N. Barnett and C. L. Cleveland, Phys. Rev. B37, 4647 (1988).

10. S. R. Phillpot and D. Wolf, Proc. MRS Symp. on "Interfacial Structure, Properties, and Design in Solids", Reno, NV 1988 (to be published); S. R. Phillpot and D. Wolf, Phil. Mag. A (to be published).

11. J. F. Lutsko, D. Wolf, S..Yip, S. R. Phillpot, and T. Nguyen, Phys. Rev. B (to be published). 